\title{
Kinky DNA in solution: Small-angle-scattering study of a nucleosome positioning sequence
}

\author{
Torben Schindler, ${ }^{1}$ Adrián González, ${ }^{2,3}$ Ramachandran Boopathi, ${ }^{4,5}$ Marta Marty Roda, ${ }^{3}$ Lorena Romero-Santacreu, ${ }^{3}$ \\ Andrew Wildes, ${ }^{2}$ Lionel Porcar, ${ }^{2}$ Anne Martel, ${ }^{2}$ Nikos Theodorakopoulos, ${ }^{6,7}$ Santiago Cuesta-López, ${ }^{3}$ Dimitar Angelov, ${ }^{4}$ \\ Tobias Unruh, ${ }^{1}$ and Michel Peyrard ${ }^{8, *}$ \\ ${ }^{1}$ Lehrstuhl für Kristallographie und Strukturphysik, Friedrich-Alexander-Universität Erlangen-Nürnberg, Staudtstr. 3, \\ D-91058 Erlangen, Germany \\ ${ }^{2}$ Institut Laue-Langevin, 71 avenue des Martyrs, CS 20156, 38042 Grenoble Cedex 9, France \\ ${ }^{3}$ ICCRAM, University of Burgos, Science and Technology Park, Plaza Misael Bañuelos, 09001 Burgos, Spain \\ ${ }^{4}$ Université de Lyon, Laboratoire de Biologie et de Modélisation de la Cellule (LBMC) CNRS/ENSL/UCBL UMR 5239, Ecole Normale \\ Supérieure de Lyon, 69007 Lyon, France \\ ${ }^{5}$ Institut Albert Bonniot, Université de Grenoble Alpes/INSERM U1209/CNRS UMR 5309, 38042 Grenoble Cedex 9, France \\ ${ }^{6}$ Theoretical and Physical Chemistry Institute, National Hellenic Research Foundation, Vasileos Constantinou 48, GR-11635 Athens, Greece \\ ${ }^{7}$ Fachbereich Physik, Universität Konstanz, D-78457 Konstanz, Germany \\ ${ }^{8}$ Université de Lyon, Ecole Normale Supérieure de Lyon, Laboratoire de Physique CNRS UMR 5672, 46 allée d'Italie, \\ F-69364 Lyon Cedex 7, France
}

(Received 30 March 2018; published 31 October 2018)

\begin{abstract}
DNA is a flexible molecule, but the degree of its flexibility is subject to debate. The commonly-accepted persistence length of $l_{p} \approx 500 \AA$ is inconsistent with recent studies on short-chain DNA that show much greater flexibility but do not probe its origin. We have performed $\mathrm{x}$-ray and neutron small-angle scattering on a short DNA sequence containing a strong nucleosome positioning element and analyzed the results using a modified Kratky-Porod model to determine possible conformations. Our results support a hypothesis from Crick and Klug in 1975 that some DNA sequences in solution can have sharp kinks, potentially resolving the discrepancy. Our conclusions are supported by measurements on a radiation-damaged sample, where single-strand breaks lead to increased flexibility and by an analysis of data from another sequence, which does not have kinks, but where our method can detect a locally enhanced flexibility due to an AT domain.
\end{abstract}

DOI: 10.1103/PhysRevE.98.042417

\section{INTRODUCTION}

Remarkable progress has been achieved since the famous publication of the DNA structure by Watson and Crick [1], but basic questions are still open. One of them was raised by Crick and Klug in 1975 [2] when they examined the ease with which duplex DNA can be deformed into a compact structure like chromatin. They suggested that DNA can form a sharp bend, which they called a kink. Kinks are not merely sharp bends due to fluctuations or a broken strand but metastable structures which can exist without a drastic distortion of the configuration of the backbone. They have been subsequently found in highly constrained environments, such as in chromatin, where other molecules force sharp bends [3,4]. However, Crick and Klug raised a more fundamental question: Could DNA kinks occur spontaneously as a result of thermal motion?

Since then this hypothesis was never confirmed and the origin of the flexibility of DNA is still debated [5]. The molecule is often described as a cylinder, or wormlike chain (WLC), that is relatively stiff with a persistence length of $l_{p} \approx 500 \AA$ [6-8]. This value is at odds with the tight packing of DNA in chromatin and with recent experiments on short-chain DNA

\footnotetext{
${ }^{*}$ To whom correspondence should be addressed: michel.peyrard@ens-lyon.fr
}

that show much shorter persistence lengths [9-12]. However, the studies are somewhat limited in that they do not probe the full conformation of the molecule and cannot identify the position or the degree of any bending.

Small-angle scattering (SAS) experiments are excellent techniques to investigate particles with linear dimensions $\sim 1-100 \mathrm{~nm}$ and are therefore well suited to studying shortchain DNA. They probe the spatial distribution of the scattering length density and are very sensitive to the overall shape and size distribution of particles. In the dilute limit, SAS probes the ensemble average of all the orientations and shapes that the particles adopt over the duration of the measurement. We have carried out measurements with both x-rays (SAXS) and neutrons (SANS) of short-chain DNA in solution, free from any molecular construct like the addition of fluorophores [12] or gold nanoparticles [13]. The principal experiments were performed with SAXS on a 145-base-pair (bp) sequence of DNA containing the "601" strong positioning sequence [14] known for easy wrapping around a histone core. The sequence, which has been investigated in structural studies of nucleosome core particles [15], is shown in Fig. 1 and is henceforth called "Widom-601." The results have been analyzed using a dynamical model. This analysis shows the presence of kinks at positions consistent with the hypothesis of Crick and Klug. As a further test, a complementary experiment has been made with a different DNA sequence, with 204 


\section{5' TGgAGAATCCCGgTGCCGAGgCCGCTCAATTGgTCGTAGACAGCTCTAGC 3' ACCTCTTAGGGCCACGGCTCCGGCGAGTTAACCAGCATCTGTCGAGATCG $57 \quad 67 \quad 88 \quad 100$ ACCGCTTAAACGCACGTACGCGCTGTCCCCCGCGTTTTAACCGCCAAGGG TGGCGAATTTGCGTGCATGCGCGACAGGGGGCGCAAAATTGGCGGTTCCC 145 \\ GATTACTCCCTAGTCTCCAGGCACGTGTCAGATATATACATCCTG $3^{\prime}$ CTAATGAGGGATCAGAGGTCCGTGCACAGTCTATATATGTAGGAC $5^{\prime}$}

FIG. 1. Sequence of the 145-base-pair DNA molecule investigated in this study. This sequence was used to build the NCP-601 nucleosome core particle investigated in Ref. [15]. The fragment with orange background is the strong positioning element, characteristic of this sequence. The TA fragments considered as possible kink positions in the model are underlined and marked in yellow.

base pairs, derived from a piece of $\lambda$ phage DNA, which has been modified to introduce a segment containing only A-T pairs from sites 94 to 125 . This introduces a domain with a higher local flexibility and some weak intrinsic curvature due to intrinsically curved elements (such as AATT). The same analysis, performed on the data collected with this control sequence did not detect kinks but the statistics of the local bending angles showed a increased average value, and increased fluctuations, in the domain where we introduced the A-T-rich segment, showing the ability of our analysis to detect detailed features of DNA flexibility with SAS.

\section{SAMPLES AND EXPERIMENTAL METHODS}

\section{A. Sample preparation}

The main sample of this study is the "Widom-601" 145 base-pair sequence of DNA shown in Fig. 1. It contains the "601" strong positioning sequence [14], known for easy wrapping around a histone core. Multiple repeats of this nucleic sequence were inserted into the EcoRV site of the pGEMT easy vector and expressed in Escherichia coli DH5 $\alpha$ cells. The fragments were excised from the vector by restriction enzyme EcoRV, followed by phenol chloroform extraction and ethanol precipitation. The excised 145-bp DNA were separated from the linearized plasmid through $5 \%$ polyacrylamide gel electrophoresis (PAGE) (prep cell-BioRad) and the purity of the sample was analyzed by $1 \%$ agarose gel electrophoresis and PAGE. This preparation method provides $100 \%$ homogeneous DNA of crystallographic-grade purity and was also applied to prepare samples for nucleosome crystallization, used in other experiments [16].The same method was used to prepare the modified $\lambda$-phage sequence shown in Fig. 2, studied in complementary experiments presented in Sec. V.

Prior to the SAXS and SANS experiments the samples were dissolved in a ${ }^{2} \mathrm{H}_{2} \mathrm{O}$ buffer containing $10 \mathrm{mM}$ Tris (pH 7.4), 0.1 mM EDTA, and $30 \mathrm{mM} \mathrm{NaCl}$ with a DNA concentration of $2.2 \mathrm{mg} / \mathrm{ml}$ for the Widom-601 sequence and $1.27 \mathrm{mg} / \mathrm{ml}$ for the modified $\lambda$-phage sequence and degassed in a partial vacuum of 0.5 bar for $3 \mathrm{~h}$ before being loaded into quartz containers for measurement. The quartz cells for SANS were rectangular with a 2-mm thickness. Quartz cylindrical capillaries with an inner diameter of $1 \mathrm{~mm}$ and a wall thickness of $10 \mu \mathrm{m}$ were used for SAXS.

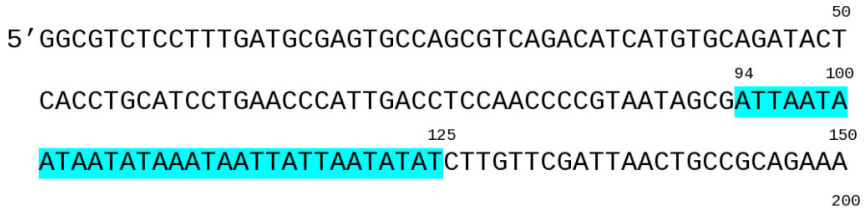

CTCTTCCAGGTCACCAGTGCAGTGCTTGATCACAGGATGCTTCCCAGGAT

GCCG $3^{\prime}$

FIG. 2. Sequence of 204 base pairs studied in a complementary experiment. In the fragment marked in blue the original sequence part of $\lambda$-phage DNA has been modified. The G-C pairs have been changed to A-T pairs to create a domain of 32 consecutive A-T pairs.

\section{B. Small-angle scattering}

Small-angle scattering of neutrons and $\mathrm{x}$ rays by dilute particles in solution probes the scattering length density of the particles. The scattering cross section is given by [17]:

$$
\frac{d \sigma}{d \Omega} \propto \frac{N}{V}\left\langle\left|\iiint_{V} \beta(\mathbf{r}, \boldsymbol{\Theta}) \exp (i \mathbf{q} \cdot \mathbf{r}) d^{3} \mathbf{r}\right|^{2}\right\rangle_{\boldsymbol{\Theta}}
$$

where the sample contains $N$ particles in volume $V$ and $\mathbf{q}$ is the momentum transfer. The scattering length density at position $\mathbf{r}$ in a particle is given by $\beta(\mathbf{r})=\rho(\mathbf{r}) \bar{b}(\mathbf{r})$, with $\rho(\mathbf{r})$ being the local atomic number density. The local mean scattering length, $\bar{b}(\mathbf{r})$, varies as a function of atom and isotope for neutrons [18] and as the Thomson scattering length multiplied by the local number of electron for $\mathrm{x}$ rays. The triangular brackets in Eq. (1) indicate that the cross section measures an ensemble average of all the orientations, $\boldsymbol{\Theta}$, and configurations that the particles have in the sample.

\section{Small-angle $x$-ray scattering}

The SAXS measurements were performed using the VAXTER instrument at the Friedrich-Alexander Universität, Germany. It uses Ga-K $\alpha 1,2$ radiation $(\lambda=1.34 \AA)$ from a GaMetalJet D2 $70-\mathrm{kV}$ x-ray source (EXCILLUM, Kista, Sweden) with 150-mm Montel optics (INCOATEC, Geesthacht, Germany). X-ray-absorbing diaphragms defined the incident beam collimation. The sample-detector distance was set to $1.5 \mathrm{~m}$, and the instrument uses a Pilatus3 $300 \mathrm{~K}$ detector. The sample temperature was controlled by an external water bath. The data were corrected for transmission and background. The scattering from a glassy carbon standard [19] was used to normalize the data to absolute scale. The resulting cross sections covered the range $0.006 \leqslant Q \leqslant 0.2 \AA^{-1}$.

\section{Small-angle neutron scattering}

The SANS measurements were performed using the D22 instrument at the Institut Laue-Langevin, France. The incident beam wavelength was set to $\lambda=6 \AA$ with $\Delta \lambda / \lambda=0.1$. The incident beam collimation was defined using neutron-absorbing diaphragms. The sample temperature was controlled by an external water bath. Data were recorded with the sample-detector distance at 5.6 and $17.6 \mathrm{~m}$, with the collimation set at $5.6 \mathrm{~m}$ and $17.6 \mathrm{~m}$, respectively, for the optimal compromise between resolution and beam flux. The 
GRASP data reduction suite (https://www.ill.eu/instrumentssupport/instruments-groups/groups/lss/grasp/home/) was used to correct the data for instrument background, empty cell, and detector efficiency and to normalize by the direct beam intensity to obtain the intensity in absolute scale. The data were then merged, buffer subtracted, and radially integrated to give the cross section in the range $0.003 \leqslant Q \leqslant 0.15 \AA^{-1}$.

\section{EXPERIMENTAL RESULTS ON THE WIDOM-601 SEQUENCE}

The SAS scattering cross section for dilute particles in solution is given by the one-dimensional integral:

$$
\frac{d \sigma}{d \Omega} \propto \int_{0}^{d_{\max }} P(r) \frac{\sin (q r)}{q r} d r,
$$

where $q$ is the momentum transfer and $d_{\max }$ is the longest dimension in the object. The pair-distribution function, $P(r)$, correlates the scattering length densities for volume elements separated by a distance $r$ within the particle and is further weighted by the contrast between the scattering length densities of the particle and the solution. The $P(r)$ therefore differs for $x$ rays and neutrons; however, for scales beyond about $40 \AA$ which do not resolve the internal structure of DNA and are only sensitive to the conformation of the molecular axis, both techniques are expected to bring the same information.

Mathematically, $P(r)$ is obtained from an inverse Fourier transform of the cross section. We used the GNOM program [20] with manually set parameters to calculate $P(r)$. The $P(r)$ derived from the SAXS data at $23{ }^{\circ} \mathrm{C}$ is shown Fig. 3(a). The measurement took $1 \mathrm{~h}$. Temperature-dependent $P(r)$ derived from the SAXS data are shown in Fig. 3(c). Two samples were measured at high temperature. For the first, labeled "short exposure," the sample was heated directly to $70^{\circ} \mathrm{C}$ and measured for $1 \mathrm{~h}$. For the second, labeled "long exposure," the data result from the final measurement of a sequence of hour-long runs at $30^{\circ} \mathrm{C}, 50^{\circ} \mathrm{C}$, and $70^{\circ} \mathrm{C}$.

The "short exposure" $P(r)$ is very similar to that at $23^{\circ} \mathrm{C}$, while the "long exposure" data show dramatic differences due to $\mathrm{x}$-ray radiation damage. At low x-ray flux, most of the damage occurs as single-strand breaks due to free radicals created by the interaction of $x$-rays with the water molecules $[21,22]$. This is likely to be exacerbated at high temperatures. In our SAXS experiment about $50 \%$ of the $x$ rays were absorbed by the solvent, and the influence of radiation damage is clearly visible.

Prior to the SAXS measurements preliminary measurements on the Widom-601 sequence had been performed with SANS as a function of temperature up to $79^{\circ} \mathrm{C}$. In situ UV absorption spectroscopy recorded during the SANS measurements showed that there was no significant thermal denaturation of double-stranded DNA up to this temperature.

The SANS measurements up to $79^{\circ} \mathrm{C}$ did show some limited changes versus temperature (Fig. 4) which could come from an increase of the thermal fluctuations, but the overall shape of $P(r)$ was only weakly modified. Radiation damage from neutrons was expected to be negligible as they are nonionizing, and the SANS data showed no strong change with exposure time.
Figure 4 compares the SAXS and SANS results. The $P(r)$ show common features. However, there are differences because SAXS is more sensitive to heavier elements as $\rho \bar{b}$ is proportional to the electron density for $\mathrm{x}$ rays. The SAXS $P(r)$ is thus dominated by the correlations between the phosphates. The neutron $P(r)$ is more sensitive to the distribution of hydrogen in the sample. Both data sets show a peak at $r \approx 20 \AA$. The peak in the x-ray data is due to the phosphates, which are on the outside of the double helix and are $20 \AA$ apart.

Due to the many protons distributed on various sites in the DNA structure, for short distances neutron scattering is well approximated by the scattering by a bulk cylinder with a diameter of $20 \AA$, which also shows a peak in $P(r)$ in the vicinity of the diameter of the cylinder. Therefore, both for SANS and SAXS, the $20 \AA$ peak appears to be signature of the diameter of the double helix.

For larger $r$ distances the differences between SAXS and SANS are expected to decrease because at this scale the differences between the atom types are no longer resolved and $P(r)$ essentially reflect the conformation of the molecules. For $r \geqslant 70 \AA$, two curves for $P(r)$ have a similar shape. They show a roughly flat region in the $70-160 \AA$ range and then decrease strongly before showing a kind of plateau in the 300-400 Å range.

\section{DATA ANALYSIS: CONFORMATION OF THE WIDOM-601 DNA MOLECULES IN SOLUTION}

We focused our analysis of the Widom-601 data on the SAXS data which provided a stronger and less noisy signal than the SANS experiments.

\section{A. Standard SAS data analysis}

As a first step we relied on standard SAS data analysis packages. The $23^{\circ} \mathrm{C}$ SAXS data were analyzed using the SASVIEW software [23] to model DNA as an homogeneous flexible cylinder, giving a persistence length of $l_{p}=97.9 \AA$. A further analysis used the Kratky-Porod model [24] which is the discrete version of the wormlike chain model generally used for long DNA molecules [6,7] and whose structure factor can be calculated exactly [25]. This calculation gave a best fit of $l_{p}=117 \AA$. Both values are much smaller than measured values for double-stranded DNA [5,26], including the generally accepted value of $l_{p} \approx 500 \AA$. They are also unrealistically small considering that the double helix has a diameter of $20 \AA$. The analysis confirms the conclusions, drawn from accurate measurements on short DNA molecules [12], that a homogeneous WLC model does not describe the flexibility of short-chain DNA.

However, it is important to stress that the persistence length obtained in these fits is actually an effective persistence length which includes both the effects of the statistical fluctuations and a contribution of the intrinsic curvature of the molecule. As pointed out by Schellmann and Harvey [27], any kind of bend, stiff or flexible, will result in a reduced persistence length and, as a result, permanent structural kinks can reduce the persistence length. 

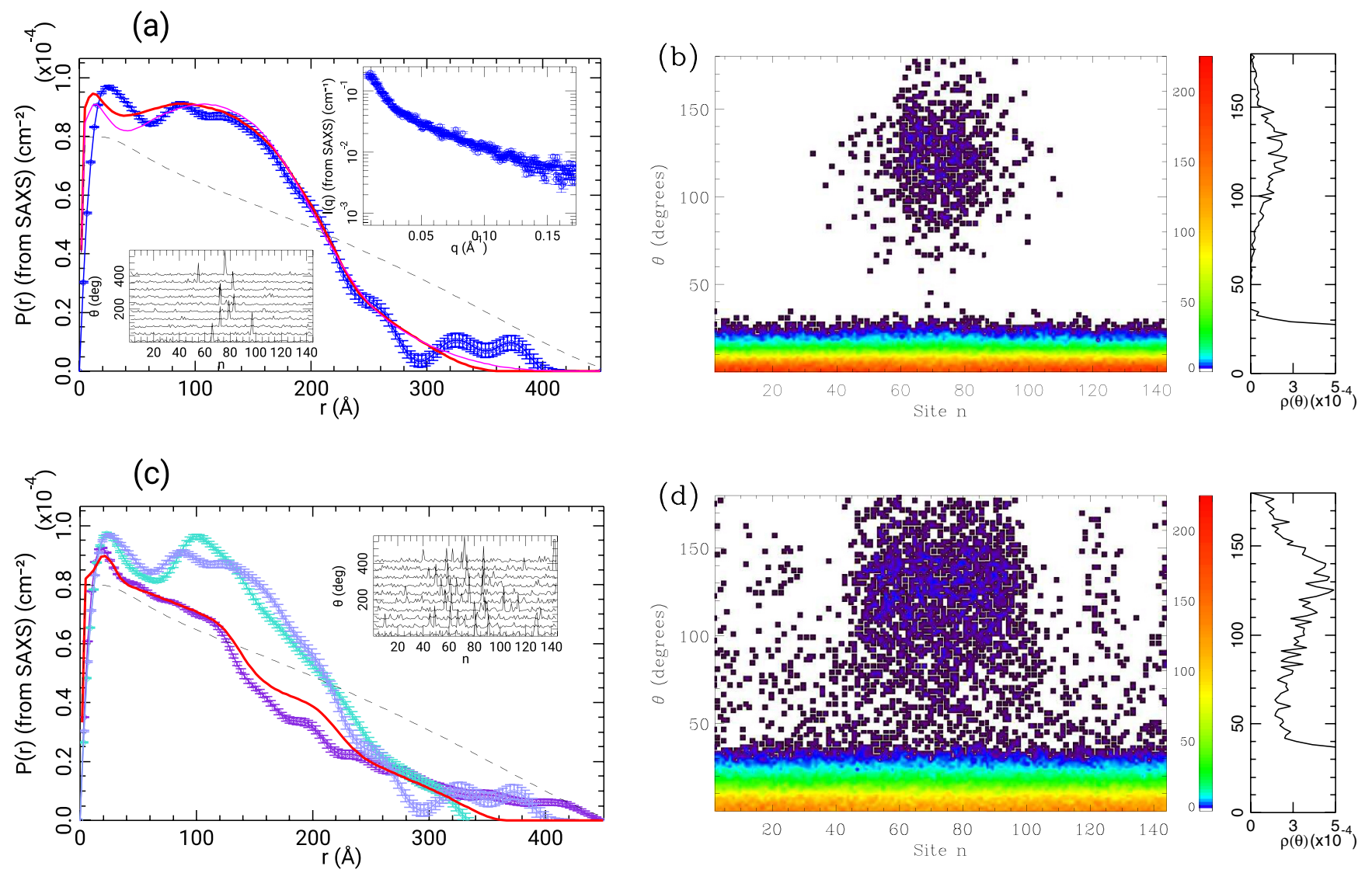

FIG. 3. Experimental results and data analysis for the Widom-601 sequence. (a) Blue: Results of the SAXS experiment at room temperature. The top right inset shows the scattered intensity $I(q)$ versus the scattering vector $q$, and the main panel shows the corresponding $P(r)$ computed by GNOM with estimated error bars. The thick red line shows the average $P(r)$ for the $10^{3}$ conformations with the best match to the experimental data among $12 \times 10^{7}$ conformations generated by Monte Carlo. Thin magenta line: $P(r)$ deduced from a Monte Carlo simulation at room temperature for the models with a kink of angle $80^{\circ}$ at site 67 . The dashed black line shows $P(r)$ for a homogeneous Kratky-Porod model having a persistence length of $500 \AA$ at room temperature $\left(K=150, \theta_{0},=0, C=0\right)$. The bottom left inset shows the values of the local bending angles for the 10 model conformations which provide the best match with the experimental $P(r)$. Successive plots are moved up by $50^{\circ}$ to limit the overlap between the curves. (b) Histogram of the bending angle against $n$ for the $10^{3}$ conformations that provide the best matching with the room temperature SAXS data. For each site of the polymer model, the left part shows the number of $\theta_{n}$ values that correspond to the value marked on the left scale (the total of these numbers for a given $n$ is equal to 1000 , the number of conformations) with a color scale shown on the right. The right part of the figure shows the fraction $\rho$ of $\theta$ angles, integrated over the whole model, which belongs to a given range of theta. The scale is truncated to $\rho_{\max }=5 \times 10^{-4}$ to better show the part of the curve which corresponds to large $\theta$ angles. (c) Dark-blue: The SAXS $P(r)$ at $70^{\circ} \mathrm{C}$ after a long exposure to x rays. The thick red curve shows the average $P(r)$ for the 1000 conformations that provide the best match with the experimental data has been obtained from a search of $70 \times 10^{7}$ conformations generated by Monte Carlo. Light blue: The SAXS $P(r)$ at room temperature [also shown in blue in panel (a)] for comparison. Turquoise: The SAXS $P(r)$ at $70^{\circ} \mathrm{C}$ with short exposure to $\mathrm{x}$ rays. The inset shows the values of the local bending angles for the 10 model conformations which provide the best match with the experimental $P(r)$ as in panel (a). (d) Same as panel (b) for the $10^{3}$ conformations that provide the best matching with long-exposure SAXS data. The color scale and the scale for $\rho$ in the right part of the plot are the same as for panel (b) to allow a quantitative comparison between the two cases.

Therefore, to understand what appears as an anomalously low persistence length, the next step was to attempt to reconstruct the main features of the particle shapes from the data. However, this is not straightforward because the knowledge of $P(r)$ is not sufficient to unambiguously determine this shape, even for the case of a linear polymer. Iterative schemes, starting from a broad and random conformational search and then progressively improving by testing likely models, have been developed. Within the ATSAS package [28], the DAMMIF program $[29,30]$ starts from the $P(r)$ given by the GNOM program and uses simulated annealing to optimize the shape of a set of dummy atoms to retrieve the shape of the scattering molecules. The program starts from a random configuration. A polymer like DNA can have a broad variety of conformations in solution and multiple runs lead to different final shapes. Nevertheless, the results give hints on the DNA conformations that fit the scattering data. When it is applied to our SAXS and SANS data for the Widom-601 sequence, this program converges either to strongly curved structures, or to branched solutions, as shown in Fig. 5. As partial denaturation of our sample is ruled out by the UV measurements, we do not expect any branching. However, if the solution actually 


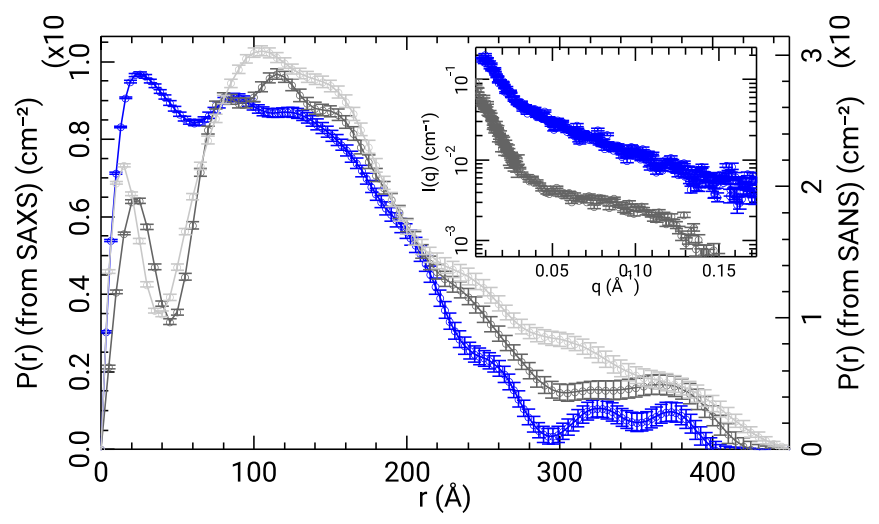

FIG. 4. Comparison of the SAXS and SANS results for the Widom-601 sequence. The SAXS data at room temperature are shown in blue and the neutron data are shown in gray (dark gray: measurements at $15^{\circ} \mathrm{C}$, light gray: measurements at $79^{\circ} \mathrm{C}$. The inset shows the scattered intensity versus the scattering vector $q$. The corresponding $P(r)$ have been obtained by GNOM. Their normalization is such that $\int P(r) d r=I(q=0)$.

contains a mixture of weakly curved molecules while others have a sharp bend, then these DAMMIF solutions could describe a superposition of two conformations because $P(r)$ represents the ensemble average over all configurations in the sample, including time-dependent fluctuations.

\section{B. Conformational search using a polymer model}

The DAMMIF results show that fluctuations must be accounted for in the modeling of the data. Moreover the ab initio shape reconstruction performed by DAMMIF does not include any a priori knowledge of the molecular properties. It is more efficient to search among a set of conformations derived from a model which takes into account some of the known features of the molecules. However, to study the flexibility of the DNA molecules, a model at the atomic scale or a coarse-grain model describing the bases would be inefficient. Instead, we
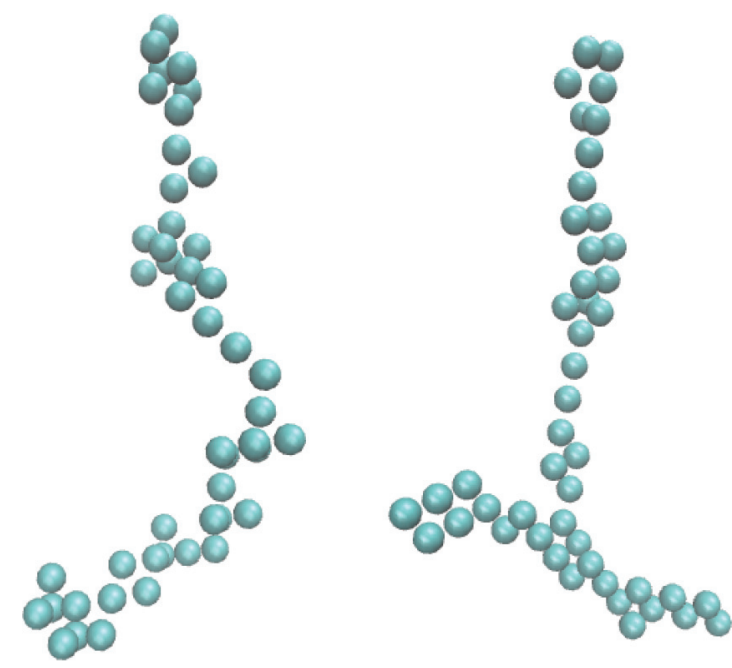

FIG. 5. Examples of the output of the DAMMIF program, showing possible optimized shapes of the DNA molecules in solution.

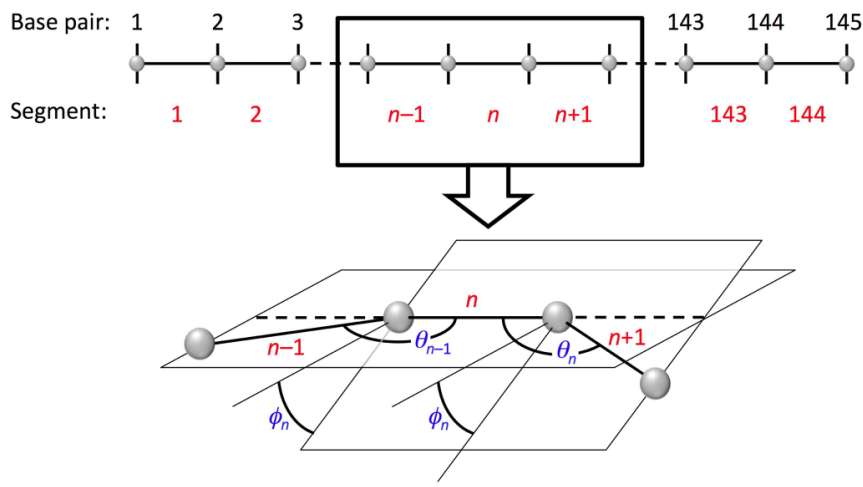

FIG. 6. Schematic showing the polymer model used for the WIDOM-601 DNA measured in the experiments. The top part of the figure shows the definition for the numbering and the lower part shows the definition of the bond and torsional rotation angles.

describe DNA with an extension of the Kratky-Porod model [24], i.e., the model, shown schematically on Fig. 6, is not concerned with the internal structure of the DNA but only with the conformations that the backbones could adopt. It consists of $N+1$ objects representing base pairs, each separated by $a=3.34 \AA$, corresponding to the base-pair distance in DNA, with bond angles that may vary at each point. The model therefore has $N$ bonds or segments. The local angle at site $n$, which connects segments $n$ and $n+1$, is defined as $\theta_{n}$, and the dihedral angle of rotation between the plane containing segments $n-1$ and $n$ and the plane containing segments $n$ and $n+1$ is defined as $\phi_{n}$.

Thermal fluctuations were accounted for by calculating the bending and torsional energy for the model expressed through its Hamiltonian. A possible permanent curvature is permitted by considering a local equilibrium value at each site, given by $\theta_{0, n}$ and $\phi_{0, n}$, which may be different from zero. The Hamiltonian is thus given by:

$H=\sum_{n=1}^{N-1} K_{n}\left[1-\cos \left(\theta_{n}-\theta_{0, n}\right)\right]+\sum_{n=2}^{N-1} C_{n}\left[1-\cos \left(\phi_{n}-\phi_{0, n}\right)\right]$,

where $K_{n}$ and $C_{n}$ are constants setting the scale of the bending and dihedral energies, respectively. Dimensionless variables were used in the calculations. Distances were measured in units of the base-pair distance $a$, and temperature was measured in energy units and the energy was expressed relative to $k_{B} T$ at room temperature, where $k_{B}$ is the Boltzmann constant, so that the properties of DNA at room temperature were obtained by setting $T=1$. Equation (3) reduces to the Kratky-Porod model [24] on setting $C_{n}=0$ and $K_{n}=K$ for all $n$. Setting $K=150$ gives $l_{p}=500 \AA$, while $K=35$ gives the previously discussed best fit to the $23^{\circ} \mathrm{C}$ data with $l_{p}=117 \AA$.

For a given model conformation, $P(r)$ was calculated by putting a unit scattering center at each base-pair position and then the calculated $P(r)$ was scaled to have the same integrated area as the data. This gives a reasonable approximation for the SAXS data for $r \gtrsim 40 \AA$, as the x-ray scattering from a base pair is dominated by its two phosphorus atoms whose contribution can be effectively mapped onto the center of the 
base pair for large $r$. For small $r$ this evaluation of $P(r)$ is a worse approximation for the SANS data which are far more sensitive to the protons.

The model with $N=144$, representing the Widom-601 sequence, was used for a broad search through the conformational space to determine the conformations which provide the best match with the experiments. At this stage of the analysis, our goal is not to design a model of the DNA molecules in solution but to extract information on the molecular conformations from the data. Therefore it is important to avoid any bias in the conformational search, which could be introduced, for instance, by assuming some $a$ priori knowledge such as sequence dependent bending angles. The bending constant was set to $K_{n}=K=150$. The torsional constant was set to $C_{n}=C=2$, which leads to a clear dominance of $\phi \approx 0$, in agreement with the torsional rigidity of DNA, but nevertheless allows large fluctuations. The equilibrium angles were set to $\theta_{0, n}=\phi_{0, n}=0$.

Up to $12 \times 10^{7}$ Monte Carlo-generated conformations were created and accepted with the probability $\exp (-H / T)$ at a temperature of $T=3$, i.e., 3 times room temperature, to widely explore the conformational space. The $P(r)$ were calculated for accepted conformations and compared to the SAXS $P(r)$ at $23^{\circ} \mathrm{C}$ by computing the standard deviations between the two functions. The best $10^{3}$ conformations were selected. They can be considered as representative of the conformations of the DNA molecules in solution so that their analysis allowed us to determine the main features of the those conformations. Their average $P(r)$ is plotted in Fig. 3(a). The agreement with the experimental $P(r)$ is satisfactory and is excellent in the region $80 \AA \leqslant r \leqslant 280 \AA$. This is the critical range to assess the shape of DNA molecules, which have a length of $484 \AA$ and a radius of gyration of about $100-150 \AA$. A calculation of the WLC with $l_{p}=500 \AA$ is also shown in Fig. 3(a) for comparison. It does not resemble the experimental $P(r)$.

Figure 3(b) shows a histogram of $\theta_{n}$ at the various sites for the best $10^{3}$ conformations. The vast majority of $\theta_{n}$ are less than $30^{\circ}$, consistent with thermal fluctuations around $\theta_{0, n}=0$. However, there is a significant concentration of large $\theta_{n}$ near the center of the molecule. The distribution is centered at $\theta_{n} \approx$ $125^{\circ}$, and it is distinct from the distribution at lower angles. Inspection of those conformations with large $\theta_{n}$ revealed that each had one, and only one, of the sites with a large angle. The insert on Fig. 3(a) shows the local bending angles for the 10 best conformations. The modeling suggests that the Widom-601 sequence has metastable states with large angles in its central region, consistent with the kinks proposed by Crick and Klug. Kinks would also help explain the branched conformations given by the DAMMIF program if the solution contains a mixture of kinked and nonkinked DNA molecules.

\section{Test of a model for kinked DNA molecules in solution}

As a blind conformational search hints that some conformations may have a permanent kink, a second stage of our analysis was to check this hypothesis. In this stage the polymer model is used in a different context. Instead of chosing a generic set of parameters to allow an unbiased scan of possible conformations, we use the results of the

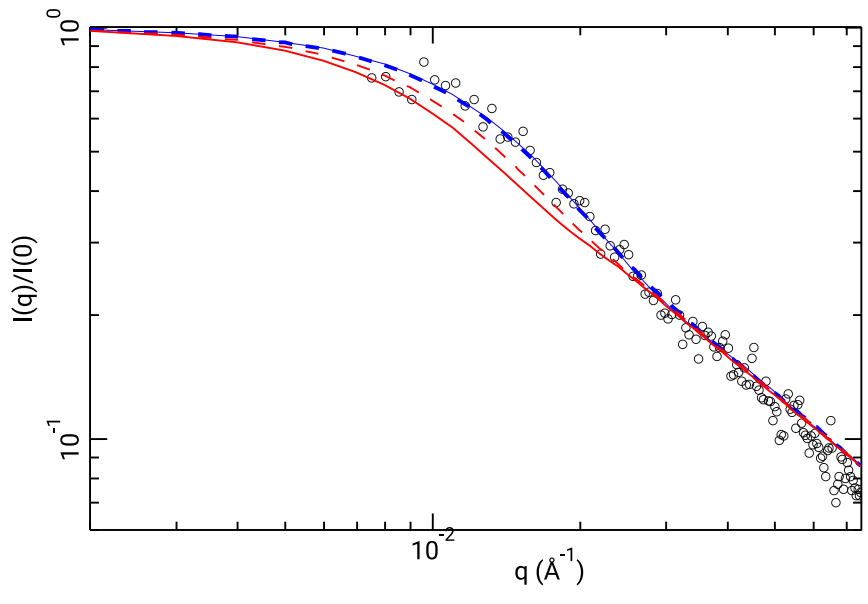

FIG. 7. Comparison between the SAXS structure factor (circles) and the structure factor of the polymer model, at room temperatures, for different parameters: (i) thin blue curve, model with a kink of $80^{\circ}$ at position 67; (ii) dashed blue curve, almost identical to the previous one, kink of $80^{\circ}$ at position 88; (iii) full red curve, Kratky-Porod model, without kink and a persistence length of $500 \AA$; (iv) dashed red curve, Kratky-Porod model, without kink and a persistence length of $224 \AA$.

conformational search to select specific parameters, and then run a Monte Carlo at room temperature ( $T=1$ in our dimensionless units) to test the agreement of the resulting $P(r)$ with the experimental results. The possible presence of a kink is tested by setting $\theta_{0, n}=\theta_{\kappa}$ for specific $n=n_{\kappa}$. Following the hypothesis of Crick and Klug, we identified TA steps in the central region and explored conformations with $70^{\circ} \leqslant \theta_{0, n}=$ $\theta_{\kappa} \leqslant 110^{\circ}$ at these sites. Inspection of Fig. 1 shows TA steps at $n_{\kappa}=47,57,67$, and 88 . The last site is of particular interest because it is part of the strong histone-positioning element characteristic of this sequence. The values for $K_{n}, C_{n}, \phi_{0, n}$, and $\theta_{0, n}$ were all maintained as for the conformational search, except for the one $\theta_{\kappa}$.

To quantitatively evaluate the different possibilities, we calculated

$$
\chi^{2}=\frac{1}{N_{r}-1} \sum_{1}^{N_{r}}\left[\frac{P_{\text {theo }}\left(r_{j}\right)-P_{\exp }\left(r_{j}\right)}{\sigma_{j}}\right]^{2},
$$

where $r_{j}$ are the points where $P_{\exp }(r)$ has been computed, $\sigma_{j}$ the experimental error at these points (given by the GNOM program), and $N_{r}$ the number of calculation points. Instead of the simpler standard deviation used for the conformational search, which is equivalent to setting $\sigma_{j}=1$ in Eq. (4), here we take into account the actual experimental errors to measure the validity of the model more accurately. The summation is restricted to $r_{j}>50 \AA$ because the polymer model, which does not take into account the diameter of the molecules, cannot be expected to properly describe the properties of the DNA molecules at very small distances.

Figure 7 compares the SAXS structure factor with the structure factor of the polymer model with a kink at one of the two TA steps closest to the center, $n_{K}=88$ (thin blue curve) or $n_{K}=67$ (dashed blue curve), and with the structure 
TABLE I. Results of Monte Carlo simulations at room temperature ( $T=1$ in reduced units) for various kink positions $n_{K}$ and angles $\theta_{K}$. The table lists the values of the $\chi^{2}$ distance between the experimental and theoretical $P(r)$ [Eq. (4)] for kinked DNA (third column). The line in bold is the case which provides the best agreement with experimental data. For nonkinked DNA, the $\chi^{2}$ distance between the theoretical and experimental probability distributions [Eq. (4)] is $\chi^{2}=22.86$.

\begin{tabular}{ccc}
\hline \hline$n_{K}$ & $\theta_{K}($ deg. $)$ & $\chi^{2}$ kink \\
\hline 88 & 95 & 2.31 \\
88 & 110 & 9.17 \\
88 & 80 & 1.26 \\
88 & 70 & 3.00 \\
67 & 95 & 3.32 \\
67 & 110 & 9.17 \\
$\mathbf{6 7}$ & $\mathbf{8 0}$ & $\mathbf{0 . 8 8}$ \\
57 & 95 & 2.14 \\
47 & 95 & 2.91 \\
\hline \hline
\end{tabular}

factor of a homogeneous WLC model, for two values of the persistence length $l_{p}=500 \AA$ and $l_{p}=224 \AA$.

The results for different kink positions and angles are given in Table I. Although such a simple polymer model cannot describe all the fine structure of the experimental results, in the range where the model applies the theoretical results fit the data within the experimental error bars in the range 50-300 $\AA$. For $r<50 \AA$ the model should be completed by taking into account the diameter of the DNA molecule and its internal structure, while for $r>300 \AA$ some extra flexibility near the ends might be necessary to take into account the fluctuations of the twist at the free boundaries.

Table I shows that the best agreement with experiments is obtained for $n_{K}=67$, although the position $\theta=88$, within the TTTAA strongly positioning sequence, gives results which are almost equally good. Our analysis cannot rule out one of the two optimal kink sites. The case of site $n_{K}=57$, which also gives good results is almost symmetric of $n_{K}=88$ with respect to the center of the molecule. For molecules in solution, at the scale of the SAXS/SANS experiments, and for the model, the two ends are indistinguishable and the 57 and 88 sites are therefore almost equivalent. Moreover, looking at the sequence of the two strands listed in Fig. 1 of the article, one can notice that, around site 57 one finds that same sequence of five base pairs as around site 88 . The best agreement with experiments is obtained for a kink angle of $80^{\circ}$, in good agreement with the kink predicted by Crick an Klug [2].

The tests were not exhaustive, but they establish that models with a kink in the DNA give far better comparisons with the experimental results than a WLC model with a sensible $l_{p}$.

\section{Analysis of the long-exposure SAXS data}

The results pointing to the possible existence of kinks for the Widom-601 sequence in solution are supported by the analysis of the SAXS data at high temperature. A conformation search with all equilibrium angles equal to zero was applied to the "long exposure" data. The search was performed at higher temperature of $T=6$ to allow for an even broader range of conformations. More than $7 \times 10^{8}$ conformations were generated in the Monte Carlo search, and the average $P(r)$ from the best $10^{3}$ conformations are shown in Fig. 3(c) with the histogram of $\theta_{n}$ vs. $n$ shown in Fig. 3(d). The results show many sites with large $\theta_{n}$ distributed more evenly across the sequence and over intermediate angles. The distribution in $\theta_{n}$ is also broader, with no gap between the low and large $\theta_{n}$ values. The plot of the local bending angles of the 10 best conformations, seen in the insert in Fig. 3(c), shows that they may have more than one large angle. The findings are consistent with radiation damage to the DNA, which creates single-strand breaks that allow local sites to be far more flexible. However, as for the search at $23^{\circ} \mathrm{C}$, a high concentration of large angles is still found around the middle of the model with an angle distribution centered at $\theta_{n} \sim 125^{\circ}$. The number of sites within this distribution is greater at $70^{\circ} \mathrm{C}$ which may represent more kinks due to increased thermal fluctuations or large bendings due to single-strand breaks, which is consistent with $\mathrm{x}$-ray radiation damage preferentially causing single-strand breaks at sites with local distortion [31], in this case due to kinks. Both observations indicate that kinks are present in Widom-601.

\section{COMPLEMENTARY EXPERIMENT WITH ANOTHER DNA SEQUENCE}

As a further test to validate our results, we have performed a complementary experiment with a DNA sequence previously studied in cyclization experiments and found to be well described by the standard WLC model of DNA [32]. This sequence, of 204 base pairs, is shown in Fig. 2. It is derived from a segment of 200 base pairs of $\lambda$-phage DNA, starting at site 29853, modified at its ends to make the polymerase chain reaction step easier in the sample preparation. This sample had been chosen for the cyclization studies because it does not have intrinsic curvature. However, in order to check the ability of our experiments and analysis to detect specific features of the DNA molecules under study, we modified the sequence locally, changing the some G-C pairs into A-T in order to create a domain of 32 consecutive A-T pairs from position 92 to position 125. Due to the larger fluctuations of the A-T pairs [33] we expect this region to have a higher flexibility. Moreover, it includes some elements, such as AATT, which have a small intrinsic curvature.

A solution with a concentration of $1.27 \mathrm{mg} / \mathrm{ml}$ was studied by SANS, using the D22 experiment at Institut Laue Langevin as described for the Widom-601 sequence. The scattering data were treated by the GNOM program to compute $P(r)$ and then we performed a computational search using the same program as for the Widom-601 sequence and the same model parameters, except for the number of nodes.

Figure 8 shows the histogram of the bending angles of the the $10^{3}$ conformations that provide the best matching with the room temperature SANS data, and its right part shows the fraction $\rho$ of $\theta$ angles, integrated over the whole model, which belongs to a given range of theta. This figure is analogous to Figs. 3(b) and 3(d) for the Widom-601 sequence, and we used the same scales for the plots to allow a quantitative comparison between the results for the two sequences. The 


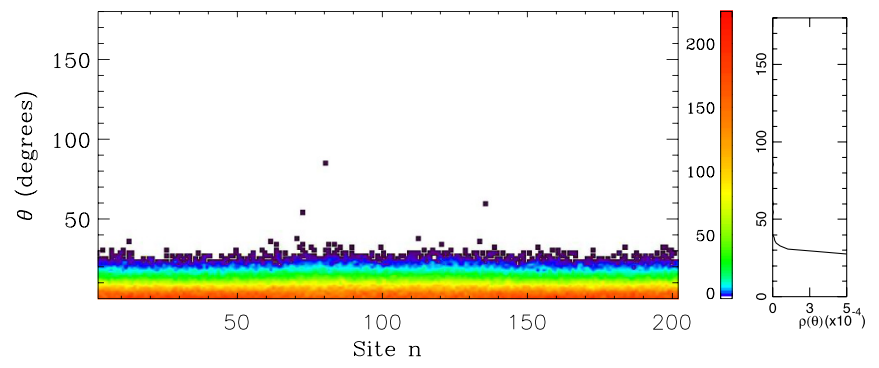

FIG. 8. Equivalent of Figs. 3(b) and 3(d) for the complementary experiment with the sequence shown in Fig. 2. Histogram of the bending angle against $n$ for the $10^{3}$ conformations that provide the best matching with the room-temperature SANS data. For each site of the polymer model, the left part shows the number of $\theta_{n}$ values that correspond to the value marked on the left scale (the total of these numbers for a given $n$ is equal to 1000, the number of conformations) with a color scale shown on the right. The right part of the figure shows the fraction $\rho$ of $\theta$ angles, integrated over the whole model, which belongs to a given range of theta. The scale is truncated to $\rho_{\max }=5 \times 10^{-4}$ to better show the part of the curve which corresponds to large $\theta$ angles.

difference is striking. For the sequence taken from $\lambda$-phage DNA we do not find the many kinked conformations detected for the nucleosome positioning sequence.

Figure 9 for the sequence taken from $\lambda$-phage DNA is equivalent to the inset on Fig. 3(a) for the Widom-601 sequence. It shows the values of the $\theta_{n}$ for the 10 conformations which provide the best match to the experimental $P(r)$ deduced from SANS measurements. Contrary to the case of the Widom-601 nucleosome positioning sequence, the bending angles do not show any sharp spike, associated to the presence of a kink.

The absence of kinks for this DNA sequence does not mean that the results of the SANS experiments are featureless. To detect the specificities of this sequence one has to look at the average bending angles $\left\langle\theta_{n}\right\rangle$ and their standard deviations $\sigma_{\theta}$. They are plotted in Fig. 10. The averages and standard deviations have been calculated over the 1000 conformations which provide the best matching with the experiments. Both the average bending and its fluctuations show maxima in

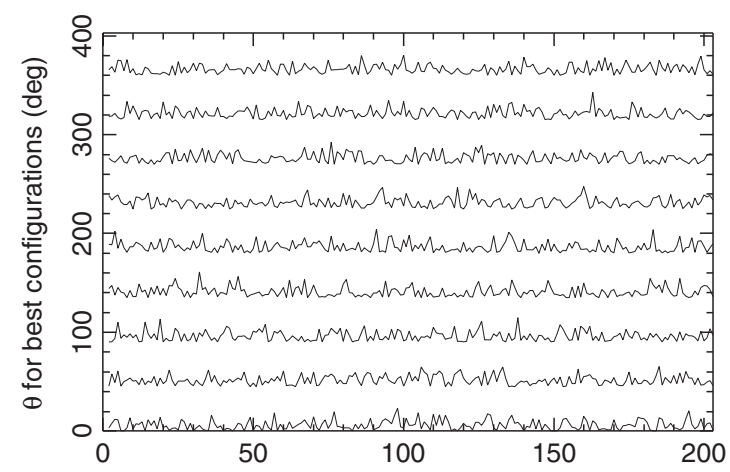

FIG. 9. Values of $\theta_{n}$ for the 10 conformations which provide the best match to the experimental $P(r)$ deduced from SANS measurements at room temperature on the sequence shown in Fig. 2. Successive plots are moved up by $50^{\circ}$ to limit the overlap between the curves.

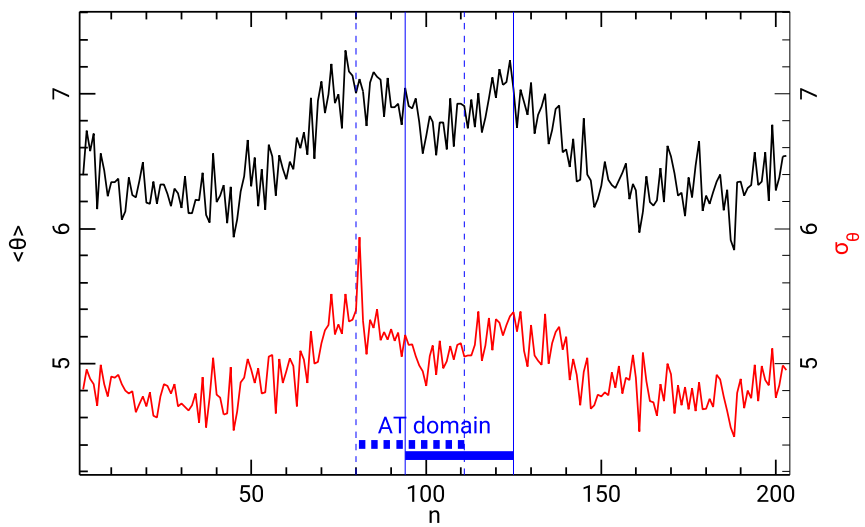

FIG. 10. Average values (black) the the local bending angles $\left\langle\theta_{n}\right\rangle$ and standard deviations (red) of the bending angles for the 1000 conformations which provide the best match to the experimental $P(r)$ deduced from SANS measurements at room temperature on the sequence shown in Fig. 2. The blue lines show the position of the $A T$ domain along the sequence, starting either from the left end of the sequence (full lines) or from the right end (dashed lines).

positions corresponding to the domain of pure A-T pairs. As the measurements do not distinguish the two ends of the molecules, the contribution of this domain on the data shows up simultaneously on the sites 94 to 125 and on 111 to 80, which correspond to its position measured from the other end of the sequence. Moreover, as we have showed using another experimental approach [33], the fluctuations of a large A-T domain influence the local conformation of DNA in its vicinity. Therefore we expect to detect the influence of the A-T domain also in its vicinity. This is exactly what the analysis of the SANS data detects, as shown in Fig. 10. The fluctuations of the A-T domain, which lead to disturbance in base stacking locally reduce the bending rigidity of double-stranded DNA. This leads to an increased standard deviation of $\theta_{n}$ and an increase in its average value due to entropic effects, which, in this case, are also reinforced by the small intrinsic curvature of some AT element. Our measurements also detect the small increase in $\left\langle\theta_{n}\right\rangle$ and $\sigma_{\theta}$ due to the free ends. Therefore Fig. 10 shows that our measurements and their analysis are able to detect fairly small effects in the conformation of DNA in solution. This reinforces our statement about the existence of kinks in the Widom-601 positioning sequence, which are large distortions which should be easier to detect.

\section{DISCUSSION}

Our experimental results on the Widom-601 sequence and their analysis clearly point to a positive answer to the question raised by Crick and Klug in 1975 [2]. Some DNA sequences can exhibit kinks, even in the absence of strong external constraints.

A first hint was provided by the analysis of the data with standard software packages developed for SAS data analysis. The fit by SASVIEW [23], as well as the Kratky-Porod model [24], lead to persistence lengths $l_{p}$ of the order of $100 \AA$, which, at a first glance appear unrealistically low. However, this would be the case if $l_{p}$ was only determined by dynamical fluctuations. But intrinsic curvature can also 
contribute to reduce the effective persistence length [27]. This effect was recently studied in details for various DNA sequences with an elaborate coarse-grain DNA model [34] and it was found that it can bring a significant contribution. Nevertheless, for the Widom-601 sequence, our experiments indicated that the effect had to be quite dramatic to reduce $l_{p}$ so much. To proceed further and determine the main features of the molecular shapes from the data, we used an extended Kratky-Porod model in two stages. First, an unbiased sampling of the conformational space using generic model parameters, without sequence dependence or intrinsic curvature, showed that the conformations providing the best match with the data exhibited a sharp, highly localized bend, in their central region. Moreover the statistics of the bending angles found a hump at large angle, separated by a gap from the large peak around $\theta=0$. This rules out a highly flexible point caused for instance by a nick because it would lead to a single-peaked distribution. The second stage, assuming a nonzero equilibrium value of the bending angle at a particular site showed that kinked conformations can indeed provide a good fit of the data. The accuracy of the SAS experiments and of our analysis cannot formally rule out a bending distribution extending over a few sites instead of a kink. This is, however, very unlikely because, owing to the very large overall bending required to fit the data, it would need several consecutive bends of $20^{\circ}$ to $30^{\circ}$ which could hardly be achieved without fully breaking the DNA structure with a high energetic cost. This is precisely because they considered such a configuration as unlikely that Crick and Klug [2] looked for an alternative. Instead, as shown by the model that they built, a kink in DNA can exist while leaving all base-pair intact and all bond distances and angles stereochemically acceptable.

Our analysis relies on the choice of a particular DNA model. We opted for a model which is as simple as possible but contains the essential features required to describe the DNA backbone. The Kratky-Porod model, often used, does not include the dihedral energy. We added this term in the Hamiltonian because it is important to control the overall shape of the polymer, which is probed in SAS experiments. Dihedral energy prevents the free rotation about the bonds, which could lead to large shape changes without affecting the bond-angle energy which enters in the Kratky-Porod model. For the first stage of our analysis, the search for conformations that best fit the data, it is important to avoid any bias in the exploration of the conformational space, and therefore the model has to be generic. The price to pay is that we have to generate a huge number of conformations $\left(\approx 10^{8}\right)$ to make sure that they include those of the molecules in solution. This price is, however, bearable because the model is sufficiently simple to allow fast calculations. In the second stage of the analysis, we try instead to design a specific model for the molecules in solution. Even within the extended Kratky-Porod model, the number of parameters that could be adjusted is very large. We minimized the number of free parameters by focusing our attention on a few equlibrium values of the bending angles, as suggested by the conformational search. Increasing the number of adjusted parameters could improve the agreement with experiments, at the risk of "overfitting" with parameters that would not be statistically significant.
Nevertheless it might be interesting to refine our analysis with improved models for DNA, such as the one used in Ref. [34]. To cover all possible conformations, the model would have to be parametrized to allow the description of kinks and not only moderate local bending. The validity of our analysis has neverteless been tested in a complementary experiment with another sequence. Using the same method of analysis we did not find kinks in this sequence but demonstrated that our approach is able to detect a fairly small effect in the conformation of DNA, validating the method. The radiation dammage, caused by a long-exposure to $\mathrm{x}$ rays, provided another, unexpected, complementary experiment. The analysis of the data is able to detect the single stand breaks, which create additional flexible points along the sequence, and also modify the probability distribution of the bending angles, by removing the gap between the small angles and the very large angles characteristic of a strong permanent bend.

Kinks in DNA are not new. They were suggested by the analysis of some cyclization experiments [10] or detected in molecular dynamics simulations of minicircles [35] and in the structure of the nucleosome core particle [15]. However, all of these examples concerned highly constrained DNA. Our results show that kinks can also exist for DNA samples in solution without any particular constraint. Therefore this peculiar DNA structure, proposed from a model building approach by Crick and Klug, could be more common than generally assumed. However, this is not a generic property of DNA. Kinks depend on the sequence and appear to be present in the nucleosome-positioning Widom-601 but not in a modified $\lambda$-phage sequence. Our findings may also help to resolve the recent debate concerning the flexibility of short-chain DNA, which has focused on differences in experimental protocols [32] but which should also consider the intrinsic properties of the DNA sequences that were investigated.

\section{ACKNOWLEDGMENTS}

We thank the 15ID-D USAXS beamline at the Advanced Photon Source, who kindly provided the glassy carbon standard sample used for the absolute normalization of the SAXS data. T.S. and T.U. gratefully acknowledge the financial support by the Deutsche Forschungsgemeinschaft (DFG) through the Cluster of Excellence Engineering of Advanced Materials (EAM) and GRK 1896 In-Situ Microscopy with Electrons, X-rays and Scanning Probes. A.G. thanks the Ph.D. program of the Institut Laue Langevin for providing the financial support for his thesis. M.M.R., L.R.S., and S.C.L. acknowledge the overall support by the Spanish Ministry of Economy, Industry and Competitiveness (BES-2013-065453, EEBB-I-2015-09973, FIS2012-38827). S.C.L. and UC-154 are grateful for the support of Junta de Castilla y Leon (Spain) Nanofibersafe BU079U16. D.A. acknowledges funding from the Agence Nationale de la Recherche through ANR-12-BSV5-0017-01 "Chrome" and ANR-17-CE11-0019-03 "Chrom3D" grants. N.T. acknowledges support by the project Advanced Materials and Devices (MIS 5002409, Competitiveness, Entrepreneurship and Innovation, NSRF 2014-2020) cofinanced by Greece and the European Regional Development Fund. 
[1] J. Watson and F. Crick, Nature 171, 737 (1953).

[2] F. Crick and A. Klug, Nature 255, 530 (1975).

[3] M. S. Ong, T. J. Richmond, and C. A. Davey, J. Mol. Biol. 368, 1067 (2007).

[4] E. Y. D. Chua, D. Vasudevan, B. W. Gabriela E. Davey, and C. A. Davey, Nucl. Acids Res. 40, 6338 (2012).

[5] J. D. Kahn, Biophys. J. 107, 282 (2014).

[6] J. Marko and E. Siggia, Macromolecules 28, 8759 (1995).

[7] J. Yan, R. Kawamura, and J. F. Marko, Phys. Rev. E 71, 061905 (2005).

[8] A. Mastroianni, D. Sivak, P. Geissler, and A. Alivisatos, Biophys. J. 97, 1408 (2009).

[9] T. E. Cloutier and J. Widom, Molecular Cell 14, 355 (2004).

[10] T. E. Cloutier and J. Widom, Proc. Natl. Acad. Sci. USA 102, 3645 (2005).

[11] C. Yuan, H. Chen, X. W. Lou, and L. A. Archer, Phys. Rev. Lett. 100, 018102 (2008).

[12] R. Vafabakhsh and T. Ha, Science 337, 1097 (2012).

[13] R. S. Mathew-Fenn, R. Das, T. D. Fenn, M. Schneiders, and P. A. B. Harbury, Science 322, 446 (2008).

[14] P. T. Lowary and J. Widom, J. Mol. Biol. 276, 19 (1998).

[15] D. Vasudevan, E. Y. D. Chua, and C. A. Davey, J. Mol. Biol. 403, 1 (2010).

[16] J. Bednar, I. Garcia-Saez, R. Boopathi, A. R. Cutter, G. Papai, A. Reymer, S. H. Syed, I. N. Lone, O. Tonchev, C. Crucifix, C. Papin, D. A. Skoufias, H. Kurumizaka, R. Lavery, A. Hamiche, J. J. Hayes, P. Schultz, D. Angelov, C. Petosa, and S. Dimitrov, Mol. Cell 66, 384 (2017).

[17] D. S. Sivia, Elementary Scattering Theory (Oxford University Press, Oxford, 2011).

[18] V. F. Sears, Neutron News 3, 26 (1992).
[19] F. Zhang, J. Ilavsky, G. G. Long, J. P. G. Quintana, A. J. Allen, and P. R. Jemian, Metall. Mat. Trans. A 41, 1151 (2010).

[20] D. Svergun, J. Appl. Cryst. 25, 495 (1992).

[21] C. von Sonntag, The Chemical Basis of Radiation Biology (Taylor \& Francis, London, 1989).

[22] R. M. Abolfath, A. C. T. van Duin, and T. Brabec, J. Phys. Chem. A 115, 11045 (2011).

[23] Sasview for small angle scattering analysis https://www.sasview.org.

[24] O. Kratky and G. Porod, Recl. Trav. Chim Pays Bas 68, 1106 (1949).

[25] N. Theodorakopoulos, Calculation of the structure factor of the heterogeneous Kratky-Porod polymer chain (2017).

[26] P. Hagerman, Ann. Rev. Biophys. Biophys. Chem. 17, 265 (1988).

[27] J. A. Schellman and S. C. Harvey, Biophys. Chem. 55, 95 (1995).

[28] M. V. Petoukhov, D. Franke, A. V. Shkumatov, G. Tria, A. G. Kikhney, M. Gajda, C. Gorba, H. D. T. Mertens, P. V. Konarev, and D. I. Svergun, J. Appl. Cryst. 45, 342 (2012).

[29] D. Svergun, Biophys. J. 76, 2879 (1999).

[30] D. Franke and D. Svergun, J. Appl. Cryst. 42, 342 (2009).

[31] J. H. Miller, J. M. Nelson, M. Ye, C. E. Swenberg, J. M. Speicher, and C. J. Benham, Int. J. Radiat. Biol. 59, 941 (1991).

[32] Q. Du, C. Smith, N. Shiffeldrim, M. Vologodskaia, and A. Vologodskii, Proc. Natl. Acad. Sci. USA 102, 5397 (2005).

[33] S. Cuesta-López, H. Menoni, D. Angelov, and M. Peyrard, Nucl. Acids Res. 39, 5276 (2011).

[34] J. S. Mitchell, J. Glowacki, A. E. Grandchamp, R. S. Manning, and J. H. Maddocks, J. Chem. Theory Comput. 13, 1539 (2017).

[35] F. Lankas, R. Lavery, and J. Maddocks, Structure 14, 1527 (2006). 